Research Article

\title{
Determinants of Preterm Birth among Mothers Who Gave Birth in Dilla University Referral Hospital, Southern Ethiopia: A Case-Control Study
}

\author{
Dagmawit Wakeyo, ${ }^{1}$ Yohannes Addisu, ${ }^{2}$ and Moges Mareg $\mathbb{D}^{1}$ \\ ${ }^{1}$ Department of Reproductive Health, School of Public Health, College of Health Science and Medicine, Dilla University, \\ Dilla, Ethiopia \\ ${ }^{2}$ School of Public Health, College of Health Science and Medicine, Dilla University, Dilla, Ethiopia \\ Correspondence should be addressed to Moges Mareg; metanmann@gmail.com
}

Received 25 June 2020; Revised 11 November 2020; Accepted 12 December 2020; Published 16 December 2020

Academic Editor: Kazim Husain

Copyright (c) 2020 Dagmawit Wakeyo et al. This is an open access article distributed under the Creative Commons Attribution License, which permits unrestricted use, distribution, and reproduction in any medium, provided the original work is properly cited.

\begin{abstract}
Globally, every year, 1.1 million newborns die due to prematurity. In Ethiopia, 320,000 preterm births occur each year; out of these, 24,400 deaths were due to preterm complications. However, there is little evidence about preterm birth in the study area. Therefore, this study provides an important direction for health professionals, health programmers, and researchers. A facility-based unmatched case-control study design was employed among 244 women (61 cases and 183 controls) who gave birth in Dilla University Referral Hospital and were selected with purposive sampling. The bivariate and multivariable logistic regression model was used to select independent predictors of preterm birth. The multivariate analysis was used, and the results were interpreted using an adjusted odds ratio at 95\% confidence interval and statistically significant level at a $P$ value less than 0.05 . A total of 240 mothers (60 cases and 180 controls) were included in the study with a $98.3 \%$ response rate. Factors like attending secondary educational and above [adjusted odd ratio $(\mathrm{aOR})=0.07(0.08-0.65)]$ and attending antenatal care $[\mathrm{aOR}=0.41(0.18$ $0.93)$ ] were protective whereas having urinary tract infection $[\mathrm{aOR}=3.6(1.1-11)]$, having human immune virus diseases $[\mathrm{aOR}=4.2(0.9-18)]$, having a history of abortion $[\mathrm{aOR}=2.3(1.1-5)]$, having a history of preterm delivery $[\mathrm{aOR}=5(1.6-15)]$, and having hypertensive disorders of pregnancy $[\mathrm{aOR}=5(1.9-13)]$ were significantly associated risk factors for preterm birth. The main determinant factors for preterm birth are having antenatal care follow-up, attending secondary education and above, hypertensive disorders of pregnancy, having HIV/AIDS, and history of abortion. This shows a need to strengthen female education; screen mothers for HIV/AIDS, urinary tract infection, and hypertension; and strengthen nutritional counseling, during ANC visits.
\end{abstract}

\section{Introduction}

Preterm birth is defined as all births before 37 completed weeks of gestation or less than 259 days since the first day of a woman's last normal menstrual period. It is classified as medically indicated (iatrogenic) and spontaneous (idiopathic) preterm birth. And according to gestational age, it is also classified as extremely preterm $(<28$ weeks), very preterm $(28$ to $<32$ weeks), and moderate to late preterm (32 to $<37$ weeks). It is the leading cause of prenatal morbidity and mortality in both developed and developing countries including Ethiopia. In most cases, preterm deliveries occur spontaneously and are associated with long-term complications in surviving infants [1].

The majority of global preterm births occur in Asia and Africa where health systems are weak and access to utilization of health services is limited [2]. The lowest rate of preterm birth occurred in Europe (6.2\%) and the highest rates occurred in Africa (11.9\%) and North America (10.6\%) [3-5]. According to different studies in Ethiopia, 
the prevalence of preterm birth is $4.4 \%$ in Gondar [6], $16.5 \%$ in Addis Ababa [7], and $25.9 \%$ in Jimma [8], whereas there is limited evidence in the study area especially related to the determinant factors of preterm birth.

The global community committed to the Sustainable Development Goals and Every Woman and Every Child initiatives to prevent and improve survival of preterm births; different interventions have been implemented which need huge financial capacity [9]. Some of the interventions include antenatal corticosteroid, antibiotic, kangaroo mother care, immediate intensive care unit service, and long-term complex health needs $[2,5]$. These interventions lead to a high economic burden for the family and the community at large through implementations of programs, policies, and strategies [8].

The national-level profile provides the most current national-level information on the status of prevention and cares for preterm birth and low birth weight in Ethiopia. More effort is needed to identify women at risk of preterm labor and support them to give birth in a health facility that can offer extra care when needed, such as support for adequate feeding with breast milk, continuous skin to skin contact, antibiotics, and antenatal corticosteroids [2]. To do this, families, communities, and health care workers must value small babies so that they receive the lifesaving care they need [5].

The preterm birth causes significant health consequences to the infant and economic costs for families and communities. In advances, prenatal and neonatal care have improved the survival of preterm infants, but those infants who do survive have a risk of developmental disabilities, health and growth problems, chronic diseases, and mortality later in life [1] than infants born at $>37$ weeks of gestation [10]. About $75 \%$ of prenatal deaths and $50 \%$ of neurological abnormalities are directly related to preterm birth [11]. The increased prevalence of medical disabilities, learning difficulties, and behavioral and psychological problems among surviving preterm infants has raised concerns that these infants may have difficulties in coping during the stage of adult life [12].

According to the Gedeo Zone maternal and child health $(\mathrm{MCH})$ report in 2017, preterm births were 122 (0.6\%); from these, $103(84 \%)$ were alive and $19(16 \%)$ were dead (Gedeo Zone health department report, 2017). This study is aimed at identifying the determinants factors of preterm birth in the Gedeo Zone, Dilla University Referral Hospital.

\section{Methods}

2.1. Study Design and Setting. A facility-based unmatched case-control study was employed from May $5^{\text {th }}$ to July $18^{\text {th }}$, 2018. The study was conducted at Dilla University Referral Hospital which is situated in Dilla town, Gedeo Zone, South Ethiopia. Dilla town is located $360 \mathrm{~km}$ away from Addis Ababa, the capital city of Ethiopia. This referral hospital has seven departments: surgical, gynecologic/obstetric, medical, radiology, dermatology, psychiatry, and pediatric wards. Taking into account the better curative services provided for, it is one of the major important destinations for patients coming from all directions of the zone, including other bounding regions like the Oromia region and Sidama and Amaro Kelle zones [13].

2.2. Population and Participants. Women who gave birth less than 37 weeks of gestation were cases, and women who gave birth greater than or equal to 37 weeks of gestation were controls. The gestational age was calculated based on the last normal menstrual period (LNMP) or ultrasound examination (for those who have early ultrasound examination). All the cases and controls were recruited during the time of child birth.

The sample size was computed using a formula for two population proportions and calculated by OpenEpi version 3.03 statistical software package by considering $23.6 \%$ of cases and $7.5 \%$ of controls exposed taken from a study done in Addis Ababa public hospitals [7]. After adjustment for $10 \%$ nonresponse rate, the final sample size for cases was 61 and for controls was 183. Finally, the total sample size becomes 244. Purposive sampling technique was used to select the study participants. Seven hundred twenty-nine mothers who gave birth in Dilla University Referral Hospital during the study period were the study population. Mothers who had serious illness (unable to communicate during data collection) and edema, which may result in defect on mid-upper arm circumference (MUAC) measurement, were excluded.

2.3. Variables of the Study. The outcome of the study was preterm birth, and the independent variables were sociodemographic characteristics (maternal age, marital status, family size, educational status, residence, occupational status, and family income), obstetrics-related variables (antenatal follow-up, history of abortion, history of still birth, history of bleeding, and medication use during pregnancy), medical condition (hypertension, diabetes mellitus, HIV/AIDS, and urinary tract infection), and maternal nutritional factor (mid-upper arm circumference (MUAC)).

2.4. Data Collection Instrument. Data collection instruments were adapted from the Ethiopia Demographic and Health Survey (EDHS) and different kinds of literature [14-17]. It is composed of sociodemographic factors, obstetric, medical condition, and maternal nutritional factors.

The outcome variable preterm birth and independent variables like sociodemographic, obstetric, and medical history were collected from the mother by using a structured questionnaire by direct interview, and data on medical factors were extracted from medical records. Gestational age was measured by clinicians either through the last normal menstrual period (LNMP) or ultrasound examination of those women who had preterm birth.

Mid-upper arm circumference (MUAC) was measured by using flexible nonstretchable tape taken at the midpoint of the left upper arm at the relaxed position without any clothing and with optimal tape tension between the acromion process on the shoulder blade and tip of olecranon process of the ulna.

The questionnaire was prepared in English and translated to the local language and then backtranslated to English 
to check the consistency. Data collection was done by trained diploma nurses. Two-day training was given for data collectors on the objectives of the study, the contents of the questionnaire, issues related to the confidentiality of the responses, and the rights of the respondents. Pretest was done at the Yirgacheffe District Hospital on 10\% (24 women), which is $24 \mathrm{~km}$ away from Dilla town. The collected data was checked daily by the principal investigator, supervisors, and data collectors to reduce incomplete data.

2.5. Data Processing, Analysis, and Presentation. First, the data were checked for their completeness and consistency and entered into OpenEpi version 3.03 exported to SPSS statistical software version 20 for analysis. Descriptive statistics were used to measure the characteristics of the study participants. Both bivariable and multivariable logistic regression analysis were computed to identify factors associated with preterm birth. Variables with a $P$ value of less than 0.25 in a bivariable analysis were considered as candidates for multivariable logistic regression. In multivariable analysis, a variable with a $P$ value of $\leq 0.05$ was considered as having statistically significant association with preterm birth. The level of the association was measured by using an odds ratio (OR) with corresponding 95\% confidence interval (CI).

2.6. Ethical Approval and Consent to Participate. The study was approved by the institutional review board (IRB) of Dilla University College of Health Science and Medicine. For the purpose of data collection from the participants, an explanation was given to them regarding to the purpose of the study, the importance of their participation, and true responses. It was also explained that the study had no connection with the individual affairs of respondents. All sampled populations were encouraged to participate in the study, while at the same time, their right not to participate was also respected. Volunteer participants signed written informed consent. For those participants less than 18 years old, we got written consent from their parents and guardians and assent from them. Women who have a preterm baby in the data collection period were counseled about nutrition and provision of care to their child.

\section{Results}

From a total of 244 women, 240 (60 cases and 180 controls) responded, and the response rate was $98.3 \%$ in both cases and controls. The case and control groups have comparable sociodemographic characteristics. The mean maternal age in both groups was 29 ( $\mathrm{SD} \pm 5.4$ ) years. In both study groups, more than $86.6 \%$ of the women were married. Among cases, $6(10 \%)$ attended tertiary education while the proportion of mothers who attended above tertiary education among controls was 56 (31\%) (Table 1).

3.1. Maternal Obstetric and Medical Characteristics. Regarding the ANC service utilization during pregnancies, 35 (58\%) of the cases and 145 (81\%) of the controls had ANC followup. Twenty-three percent of the cases and $31 \%$ of the controls had 4 or more ANC visits during pregnancy. Around $27 \%$ of the cases and $11 \%$ of the controls had hypertensive disorders
TABLE 1: Sociodemographic characteristics of women who gave birth at Dilla University Referral Hospital, Gedeo Zone, Southern Ethiopia, May 5-July 18, $2018(n=240)$.

\begin{tabular}{|c|c|c|}
\hline \multirow[b]{2}{*}{ Variable } & \multicolumn{2}{|c|}{ Study subjects } \\
\hline & $\begin{array}{c}\text { Case }(n, \%) \\
n=60\end{array}$ & $\begin{array}{c}\text { Control }(n, \%) \\
n=180\end{array}$ \\
\hline \multicolumn{3}{|l|}{ Age } \\
\hline $15-24$ & $14(23)$ & $29(16)$ \\
\hline $25-34$ & $39(65)$ & $108(60)$ \\
\hline$\geq 35$ & $7(12)$ & $43(24)$ \\
\hline \multicolumn{3}{|l|}{ Ethnicity } \\
\hline Gedeo & $32(53.3)$ & $74(41)$ \\
\hline Oromo & $11(18.3)$ & $37(20.5)$ \\
\hline Amhara & $3(5)$ & $19(10.5)$ \\
\hline Other $^{\mathrm{a}}$ & $14(23.4)$ & $50(28)$ \\
\hline \multicolumn{3}{|l|}{ Marital status } \\
\hline Married & $52(86.6)$ & $174(96.6)$ \\
\hline Divorce and widowed & $8(13.4)$ & $6(3.4)$ \\
\hline \multicolumn{3}{|c|}{ Educational status of the mother } \\
\hline Cannot read and write & $36(60)$ & $53(29)$ \\
\hline Primary education & $6(10)$ & $12(7)$ \\
\hline Secondary education & $12(20)$ & $59(33)$ \\
\hline Tertiary and above & $6(10)$ & $56(31)$ \\
\hline \multicolumn{3}{|l|}{ Residences } \\
\hline Urban & $29(48.3)$ & $77(42.7)$ \\
\hline Rural & $31(51.7)$ & $103(57.2)$ \\
\hline \multicolumn{3}{|c|}{ Monthly income (Ethiopian birr) } \\
\hline$<600 \mathrm{ETB}$ & $25(42)$ & $59(33)$ \\
\hline$\geq 600 \mathrm{ETB}$ & $35(58)$ & $121(67)$ \\
\hline
\end{tabular}

Farmer, daily laborer, student. ${ }^{\text {a}}$ Sidama, Wolita, and Gurage.

of pregnancy (HDP). About $11(18 \%)$ of the cases and $8(4 \%)$ of the controls have a history of preterm birth. Nine (15\%) of the cases and $5(2.7 \%)$ of the controls were HIV positive. Thirty-eight percent of the cases and $23.3 \%$ of the controls had a history of abortion in the previous pregnancy. Regarding medication use, $20 \%$ of the cases and $14.4 \%$ of the controls use medication during pregnancy. Fifty-eight percent of the cases and $52.8 \%$ of the controls had more than 5 family sizes (Table 2).

3.2. Determinants of Preterm Birth. Variables that have a $P$ value of less than 0.25 were moved to multivariable logistic regression analysis to determine the predictors of preterm birth. Based on the final multivariable analysis model, variables that were identified as independent predictors of preterm birth were as follows: attending education secondary and above level, having ANC follow-up, HIV-positive serostatus, having urinary tract infection, history of abortion, history of preterm birth, and hypertensive disorder of pregnancy.

In this finding, women who had attended tertiary education were $93 \%$ less likely to have preterm birth as compared 
TABle 2: Obstetric and medical characteristics of women who gave birth at Dilla University Referral Hospital, Gedeo Zone, Southern Ethiopia, May 5-July 18, $2018(n=240)$.

\begin{tabular}{|c|c|c|}
\hline \multirow[b]{2}{*}{ Variable } & \multicolumn{2}{|c|}{ Study subjects } \\
\hline & $\begin{array}{c}\text { Cases } \\
\text { (number \%) }\end{array}$ & $\begin{array}{c}\text { Controls } \\
\text { (number \%) }\end{array}$ \\
\hline \multicolumn{3}{|c|}{ Attending antenatal care (ANC) } \\
\hline Yes & $35(58)$ & $145(81)$ \\
\hline No & $25(42)$ & $35(19)$ \\
\hline \multicolumn{3}{|c|}{ Number of ANC visits } \\
\hline 1 times & $11(18)$ & $22(12)$ \\
\hline 2-3times & $10(16)$ & $67(37)$ \\
\hline$\geq 4$ times & $14(23)$ & $56(31)$ \\
\hline \multicolumn{3}{|l|}{ Family size } \\
\hline $1-2$ & $1(2)$ & $2(1.2)$ \\
\hline $3-4$ & $24(40)$ & $83(46)$ \\
\hline$\geq 5$ & $35(58)$ & $95(52.8)$ \\
\hline \multicolumn{3}{|c|}{ Hypertensive disorders of pregnancy } \\
\hline Yes & $16(27)$ & $20(11)$ \\
\hline No & $44(73)$ & $160(89)$ \\
\hline \multicolumn{3}{|c|}{ UTI (urinary tract infection) } \\
\hline Yes & $11(18.3)$ & $13(7.2)$ \\
\hline No & $49(81.7)$ & $167(92.8)$ \\
\hline \multicolumn{3}{|c|}{ HIV/AIDS serostatus } \\
\hline Positives & $9(15)$ & $5(2.7)$ \\
\hline Not positive & $51(85)$ & $175(97.3)$ \\
\hline \multicolumn{3}{|c|}{ History of preterm birth } \\
\hline Yes & $11(18)$ & $8(4)$ \\
\hline No & $49(82)$ & $172(96)$ \\
\hline \multicolumn{3}{|c|}{ History of abortion } \\
\hline Yes & $23(38)$ & $42(23.3)$ \\
\hline No & $37(62)$ & $138(76.7)$ \\
\hline \multicolumn{3}{|c|}{ Medication use during pregnancy } \\
\hline Yes & $10(20)$ & $26(14.4)$ \\
\hline No & $50(80)$ & $154(85.5)$ \\
\hline
\end{tabular}

to those who had not attended education $(\mathrm{OR}=0.07, \mathrm{CI}=$ $0.08-0.65)$. Women who had ANC follow-up were $59 \%$ less likely to have preterm birth as compared to those who had no ANC follow-up ( $\mathrm{OR}=0.41, \mathrm{CI}=0.18-0.93]$. Women who had hypertension disorder during pregnancy were 5 times more likely to have preterm birth as compared to those who had no hypertension disorder during pregnancy $(\mathrm{OR}=5, \mathrm{CI}=1.9-13)$. Women who had urinary tract infection during pregnancy were 3.6 times more likely to have preterm birth as compared to those who have no urinary tract infection during pregnancy $(\mathrm{OR}=3.6, \mathrm{CI}=1.1-11)$. HIVpositive women were 4.2 times more likely to have a preterm baby as compared to their counterparts $(\mathrm{OR}=4.2, \mathrm{CI}=0.9$ -18). Those women who had a history of preterm birth were 5 times more likely to have preterm birth as compared to their counterparts $(\mathrm{OR}=5, \mathrm{CI}=1.6-15)$. Women who had a history of abortion were 2.3 times more likely to have preterm birth as compared to those who had no history of abortion $(\mathrm{OR}=2.3, \mathrm{CI}=1.1-5)($ Table 3$)$.

\section{Discussion}

This study was an unmatched case-control study on 240 mothers who gave birth at Dilla University Referral Hospital intending to identify the determinant of preterm births. According to this study, the variables like educational status, receiving antenatal care, having hypertensive disorders of pregnancy, HIV/AIDS-positive serostatus, having urinary tract infection, having a history of preterm birth, and history of abortion have a statistically significant association with preterm birth.

Women who had attended tertiary and above education were $93 \%$ less likely to have preterm birth as compared to those who cannot read and write. This finding is in agreement with a study done in Jimma [8]; Bengal, India [18]; and Bangladesh [19]; women who had tertiary and above education had a low risk of preterm delivery as compared to their counterparts. This might be because when a woman becomes more educated, she will have better access to information, knowledge on different health problems, and prevention skills.

The other factor found to be associated with preterm birth in this study was ANC visit. Mothers who had ANC visit had a 59\% decreased risk of preterm birth than mothers who had no ANC visit. This finding is supported by a study done in Debre Markos [20]; Bengal, India [18]; Tanzania [21]; Brazil [22]; and Bangladesh [19]. This might be because when the mothers have an ANC visit, they may be counseled by health professionals about early identification of risk factors, signs and symptoms, diagnosis, and treatment of preterm births in the health facilities.

This study revealed that women who had urinary tract infections during pregnancy were 3.6 times more likely to have preterm birth as compared to those who had no urinary tract infection during pregnancy. This result agreed with the study done in Tanzania [21] and Kenya [23]. Urinary tract infections can weaken the membranes of the amniotic sac around the baby. This could lead to premature rupture of the membranes and preterm labor [14].

The history of abortion is another significant risk factor associated with preterm birth. Women who had a history of abortion were 2.3 times more likely to have preterm birth as compared to those who had no history of abortion. This finding is similar to the studies conducted in Jimma [8] and Kenya [23]. The fact that abortion increases the risk of preterm birth is that surgical evacuation of the uterus mechanically stretches the cervix which predisposes such mothers to preterm birth in consecutive pregnancies [24].

Women who had a history of preterm birth were 5 times more likely to have preterm birth as compared to those who had no history of preterm birth. This finding agreed with studies done in Jimma [8]; Western Maharashtra [25]; Bengal, India [18]; and Kenya [23]. The mechanism for this has not been well understood; however, the likelihood of such 
TABle 3: Multivariate analysis for determinants of preterm birth among mothers who gave birth at Dilla University Referral Hospital, Southern Ethiopia, May 5-July 18, $2018(n=240)$.

\begin{tabular}{|c|c|c|c|c|}
\hline \multirow{2}{*}{ Variable } & \multicolumn{2}{|c|}{ Study subjects } & \multirow{2}{*}{ COR $(95 \% \mathrm{CI})$} & \multirow{2}{*}{$\operatorname{AOR}(95 \% \mathrm{CI})$} \\
\hline & Cases $(n, \%)$ & Controls $(n, \%)$ & & \\
\hline \multicolumn{5}{|c|}{ Educational status of mother } \\
\hline Illiterate & $36(60)$ & $53(29)$ & 1 & 1 \\
\hline Primary education & $6(10)$ & $12(7)$ & $0.73(0.25-2.14)$ & $0.59(0.16-2.1)$ \\
\hline Secondary education & $12(20)$ & $59(33)$ & $0.29(0.14-0.63)$ & $0.2(0.07-0.53)^{* *}$ \\
\hline Tertiary and above & $6(10)$ & $56(31)$ & $0.16(0.06-0.4)$ & $0.07(0.08-0.65)^{* *}$ \\
\hline \multicolumn{5}{|c|}{ Occupation of the mother } \\
\hline Housewife & $35(58)$ & $60(33)$ & 1 & 1 \\
\hline Merchant & $13(22)$ & $57(32)$ & $0.39(0.18-0.81)$ & $0.8(0.32-1.7)$ \\
\hline Employee & $6(10)$ & $48(27)$ & $0.21(0.083-0.55)$ & $3.2(0.35-30)$ \\
\hline Other* & $6(10)$ & $15(8)$ & $0.68(0.24-1.9)$ & $1.9(0.5-7)$ \\
\hline \multicolumn{5}{|c|}{ Monthly income (Ethiopian birr) } \\
\hline$<600$ ETB & $25(42)$ & $59(33)$ & $1.4(0.8-2.6)$ & $0.9(0.47-2)$ \\
\hline$\geq 600 \mathrm{ETB}$ & $35(58)$ & $121(67)$ & 1 & \\
\hline \multicolumn{5}{|l|}{ Antenatal care (ANC) } \\
\hline Yes & $35(58)$ & $145(81)$ & $0.33(0.18-0.63)$ & $0.41(0.18-0.93)^{* *}$ \\
\hline No & $25(42)$ & $35(19)$ & 1 & 1 \\
\hline \multicolumn{5}{|c|}{ Hypertension disorder of pregnancy } \\
\hline Yes & $16(27)$ & $20(11)$ & $2.9(1.3-6)$ & $5(1.9-13)^{* *}$ \\
\hline No & $44(73)$ & $160(89)$ & 1 & 1 \\
\hline \multicolumn{5}{|l|}{ Urinary tract infection } \\
\hline Yes & $11(18.3)$ & $13(7.2)$ & $2.8(1.2-6.8)$ & $3.6(1.1-11)^{* *}$ \\
\hline No & $49(81.7)$ & $167(92.8)$ & 1 & 1 \\
\hline \multicolumn{5}{|l|}{ HIV/AIDS serostatus } \\
\hline Positives & $9(15)$ & $5(2.7)$ & $6.1(1.98-19.2)$ & $4.2(0.9-18)^{* *}$ \\
\hline Not positive & $51(85)$ & $175(97.3)$ & 1 & 1 \\
\hline \multicolumn{5}{|l|}{ History of preterm birth } \\
\hline Yes & $11(18)$ & $8(4)$ & $4.8(1.8-12)$ & $5(1.6-15)^{* *}$ \\
\hline No & $49(82)$ & $172(96)$ & 1 & 1 \\
\hline \multicolumn{5}{|l|}{ History of abortion } \\
\hline Yes & $23(38)$ & $42(23.3)$ & $2(1.09-3.8)$ & $2.3(1.1-5)^{* *}$ \\
\hline No & $37(62)$ & $138(76.7)$ & 1 & 1 \\
\hline \multicolumn{5}{|l|}{ MUAC $(\mathrm{cm})$} \\
\hline$<23$ & $34(57)$ & $69(38)$ & 1 & 1 \\
\hline $23-35$ & $23(38)$. & $84(47)$ & $0.55(0.3-1.03)$ & $0.54(0.25-1.1)$ \\
\hline$>25$ & $3(5)$ & $27(15)$ & $0.22(0.064-0.79)$ & $0.2(0.046-0.96)^{* *}$ \\
\hline
\end{tabular}

Note: *farmer, daily laborer, and student; ${ }^{* *}$ statistical significance $P<0.05$.

experience among the women with prior spontaneous labor as well as those with inducing preterm birth is rising [10].

Another determinant of preterm birth is hypertension during pregnancy. Women who had hypertension during pregnancy were 5 times more likely to have preterm birth as compared to those who had no hypertension during pregnancy. This study is in agreement with the study done in Gondar [6], Ghana [26], Kenya [23], and Nigeria [11]. This might be because complications of pregnancy-induced hypertension can cause vascular damage to the placenta or iatrogenesis due to the severity of hypertension or its complications. This in turn induces the oxytocin receptors, which results in preterm labor and delivery, [12].

Being HIV/AIDS positive is also significantly associated with preterm birth. Women who are HIV positive were 4.2 times more likely to have preterm birth as compared to their 
counterparts. This finding is similar to studies done in Addis Ababa [7] and Tanzania [21] where women with infection during pregnancy were more likely to deliver a preterm baby than their counterparts. This might be due to the effect of antiretroviral drugs, and the reduced immunity of women is a risk factor for preterm birth [11].

Finally the limitation of the study was recall bias, being a case control study; it can only tell us the presence of association but not causal relationship. To alleviate such recall biases, the data collectors cross-checked the data from medical records of the participants.

\section{Conclusion}

This study showed the determinant factors like education level secondary and above and having antenatal care visit were protective factors, whereas having hypertension during pregnancy, HIV/AIDS-positive serostatus, having urinary tract infection, history of preterm birth, and history of abortion were risk factors for preterm birth. This shows a need for strengthening of female education; early screening and treatment of maternal HIV/AIDS, urinary tract infection, and hypertension; strengthening of antenatal care follow-up; nutritional counseling during ANC visit; and prevention of the occurrence of abortion is recommended to reduce the preterm birth.

\section{Data Availability}

All the data included in the manuscript can be accessed from the corresponding author upon request via metanmann@gmail.com.

\section{Conflicts of Interest}

The authors declare that there is no conflict of interest regarding the publication of this paper.

\section{Authors' Contributions}

All the authors contributed from proposal development to data analysis and preparation of the article, gave final approval of the version to be published, and agree to be accountable for all aspects of the work.

\section{Acknowledgments}

We would like to express our deepest gratitude to Dilla University for the ethical clearance. Our gratitude also extends to the staff of Dilla University Referral Hospital for their collaboration. We would like to thank the data collectors, supervisors, all study participants, and others who participated directly or indirectly and who spent their fruitful time for the accomplishment of this study.

\section{References}

[1] S. Beck, D. Wojdyla, L. Say et al., "The worldwide incidence of preterm birth: a systematic review of maternal mortality and morbidity," Bulletin of the World Health Organization, vol. 88 , pp. 31-38, 2010.
[2] M. V. Kinney, K. J. Kerber, R. E. Black et al., "Sub-Saharan Africa's mothers, newborns, and children: where and why do they die?," PLoS Medicine, vol. 7, no. 6, article e1000294, 2010.

[3] M. Lukasse, A. Helbig, J. Š. Benth, and M. Eberhard-Gran, "Antenatal maternal emotional distress and duration of pregnancy," PLoS One, vol. 9, no. 7, article e101682, 2014.

[4] M. Gladstone, C. Oliver, and N. Van den Broek, "Survival, morbidity, growth and developmental delay for babies born preterm in low and middle income countries-a systematic review of outcomes measured," PLoS One, vol. 10, no. 3, article e0120566, 2015.

[5] M. C. Smid, E. M. Stringer, and J. S. Stringer, "A worldwide epidemic: the problem and challenges of preterm birth in low-and middle-income countries," American Journal of Perinatology, vol. 33, no. 3, pp. 276-289, 2016.

[6] K. Gebreslasie, "Preterm birth and associated factors among mothers who gave birth in Gondar town health institutions," Advances in Nursing, vol. 2016, 5 pages, 2016.

[7] A. Deressa, A. Cherieb, and B. Negash, "Factors associated with induced preterm birth and its immediate outcome in Addis Ababa public hospitals, Ethiopia," Neonatal and Pediatric Medicine, vol. 4, no. 170, p. 2, 2018.

[8] I. Bekele, T. Demeke, and K. Dugna, "Prevalence of preterm birth and its associated factors among mothers delivered in Jimma University specialized teaching and referral hospital, Jimma Zone, Oromia Regional State, South West Ethiopia," Journal of Women's Health Care, vol. 6, no. 1, 2017.

[9] B. T. Soon, The Global Action Report on Preterm Birth, World Health Organization, Geneva, 2012.

[10] W. Belaynew, A. Teumay, G. Getachew, and K. Mohamed, "Effects of inter pregnancy interval on preterm birth and associated factors among postpartum mothers who gave birth at Felege Hiwot referral hospital," World Journal of Pharmaceutical Sciences, vol. 4, no. 4, pp. 12-25, 2015.

[11] G. R. Alexander, "Prematurity at birth: determinants, consequences and geographic variation," Preterm birth: Causes, consequences, and prevention, pp. 604-644, 2007.

[12] J. M. Moutquin, "Classification and heterogeneity of preterm birth," BJOG: An International Journal of Obstetrics \& Gynaecology, vol. 110, pp. 30-33, 2003.

[13] C. G. Victora, J. H. Requejo, A. J. Barros et al., "Countdown to 2015: a decade of tracking progress for maternal, newborn, and child survival," The Lancet, vol. 387, no. 10032, pp. 2049-2059, 2016.

[14] S. K. Sebayang, M. J. Dibley, P. J. Kelly, A. V. Shankar, A. H. Shankar, and SUMMIT Study Group, "Determinants of low birthweight, small-for-gestational-age and preterm birth in Lombok, Indonesia: analyses of the birthweight cohort of the SUMMIT trial," Tropical Medicine \& International Health, vol. 17, no. 8, pp. 938-950, 2012.

[15] D. Woldesenbet, Association of maternal anthropometrics with preterm birth and determine other risk factors for preterm birth among mothers who delivered in Gandhi Memorial Hospital and Tikur Anbessa Hospital, 2016/17, Addis Abeba Universty, 2017.

[16] H. G. Mengesha, W. T. Lerebo, A. Kidanemariam, G. Gebrezgiabher, and Y. Berhane, "Pre-term and post-term births: predictors and implications on neonatal mortality in Northern Ethiopia," BMC Nursing, vol. 15, no. 1, article 48, 2016. 
[17] EDHS, "Demographic and health survey 2016: key indicators report," The DHS Program ICF, 2016.

[18] R. K. Rai, C. R. Sudfeld, A. Barik, W. W. Fawzi, and A. Chowdhury, "Sociodemographic determinants of preterm birth and small for gestational age in rural West Bengal, India," Journal of Tropical Pediatrics, vol. 65, pp. 537-546s, 2019.

[19] A. Rahman, M. Rahman, J. Pervin et al., "Time trends and sociodemographic determinants of preterm births in pregnancy cohorts in Matlab, Bangladesh, 1990-2014," BMJ Global Health, vol. 4, article e001462, 2019.

[20] T. Bekele, A. Amanon, and K. Gebreslasi, "Pre-term birth and associated factors among mothers who gave birth in Debremarkos town health institutions, 2013 institutional based cross sectional study," Gynécologie et Obstétrique, vol. 5, no. 5, pp. 292-297, 2015.

[21] R. M. Zack, J. Golan, S. Aboud, G. Msamanga, D. Spiegelman, and W. Fawzi, "Risk factors for preterm birth among HIVinfected Tanzanian women: a prospective study," Obstetrics and Gynecology International, vol. 2014, 9 pages, 2014.

[22] E. M. Ferraz and R. H. G. A. T. M. Cunha, "Determinants of preterm delivery and intrauterine growth retardation in North-East Brazil," International Journal of Epidemiology, vol. 19, no. 1, pp. 101-108, 1990.

[23] O. T. Okube and L. M. Sambu, "Determinants of preterm birth at the postnatal ward of Kenyatta National Hospital, Nairobi, Kenya," Open Journal of Obstetrics and Gynecology, vol. 7, no. 9, p. 973, 2017.

[24] W. Yuan, A. M. Duffner, L. Chen, L. P. Hunt, S. M. Sellers, and A. L. Bernal, "Analysis of preterm deliveries below 35 weeks' gestation in a tertiary referral hospital in the UK. A casecontrol survey," BMC Research Notes, vol. 3, no. 1, article 119, 2010.

[25] S. A. Shubhada, S. V. Kambale, and B. D. Phalke, "Determinants of preterm labour in a rural medical college hospital in western Maharashtra," Nepal Journal of Obstetrics and Gynaecology, vol. 8, no. 1, pp. 31-33, 2013.

[26] K. Adu-Bonsaffoh, C. Gyamfi-Bannerman, S. A. Oppong, and J. D. Seffah, "Determinants and outcomes of preterm births at a tertiary hospital in Ghana," Placenta, vol. 69, pp. 62-67, 2019. 